\title{
REFORMA DEL PRESIDENCIALISMO: EL CASO DE LA JEFATURA DE GABINETE DE MINISTROS EN ARGENTINA (1995-2007)*
}

\section{Ricardo Gamboa}

Este artículo analiza la Jefatura de Gabinete de Ministros (JGM) en Argentina entre 1995 y 2007 con el fin evaluar su aporte como eventual mecanismo de "atenuación" del sistema presidencial argentino. Del estudio se concluye, primero, que el diseño institucional de la JGM no es conducente a atenuar el presidencialismo, sino que más bien apunta a eventualmente desconcentrar el poder presidencial. Segundo, se señala que en la práctica la JGM no cumple esa función dada la continua modificación de su estructura y atribuciones y la diferente posición que los presidentes le han asignado. Y tercero, que por su diseño no es un mecanismo adecuado para superar la "rigidez" del presidencialismo.

Palabras clave: Argentina; presidencialismo; gobierno; instituciones sistema político.

Recibido: junio 2009.

Ricardo Gamboa. Abogado, Universidad Católica de Chile. Doctor en Ciencia Política, Universität Tûbingen. Actualmente es profesor asistente del Instituto de Estudios Internacionales de la Universidad de Chile.

* Este artículo forma parte del proyecto Fondecyt 1070868. 


\section{Introducción}

$\mathrm{D}$

esde que Linz (1990) planteó su visión crítica sobre el presidencialismo se ha desarrollado un profundo y fructífero debate académico sobre sus virtudes y debilidades. Al mismo tiempo, en distintos países de América Latina se ha discutido extensamente, a nivel de dirigentes y autoridades políticas, la necesidad de introducir modificaciones en el sistema institucional. Fruto de ello, en las últimas dos décadas diversos países de la región han modificado las reglas que estructuran sus respectivos presidencialismos (Munck, 2004). Si bien ninguno de los países en que se ha modificado el sistema presidencial ha optado por establecer directamente un sistema parlamentario ${ }^{1}$, sobresale el hecho de que, como indica Negretto (2009), no hay una tendencia única en los procesos de reforma.

En efecto, por un lado, algunas reformas han procurado expandir el poder presidencial en ciertas esferas, ya sea por la vía de ampliar sus poderes legislativos o de permitir su reelección ${ }^{2}$. Por otro lado, distintas reformas han apuntado a "atenuar el presidencialismo" mediante normas que buscan un mayor equilibrio entre el presidente y el congreso, o que otorgan mayores poderes de control al congreso respecto del presidente. De esta manera, algunas reformas se han propuesto aumentar el poder de control del congreso sobre los ministros mediante el fortalecimiento de los mecanismos de interpelación (por ejemplo,

${ }^{1}$ Esto no implica decir que el tema no se haya discutido a nivel de actores políticos. De hecho, en la década de 1980 se conocieron distintas propuestas, especialmente en Argentina y Brasil, para introducir elementos "de tipo parlamentario" en sus sistemas, como la responsabilidad política del jefe de gobierno ante el parlamento (Negretto, 2009: 47).

${ }^{2}$ Según Negretto (2009:49) de 18 reformas al presidencialismo introducidas en América Latina desde 1978, el 67\% de ellas aumentaron el poder presidencial en materia legislativa. En general, ellas consisten en entregar mayores facultades a los presidentes en la discusión del presupuesto, permitirles invocar la urgencia en el tratamiento de las leyes, e incluso otorgarles la facultad de emitir, vía decreto, normas de rango legal. En el caso de la reelección, aun cuando en ningún país se ha introducido la reelección indefinida, en varios se han introducido normas que la permiten para el período siguiente (por ejemplo, Argentina en 1994; Brasil en 1998; Venezuela en 1999 y Colombia en 2005). La reelección presidencial inmediata, aun cuando no implique aumentar los poderes formales del presidente, fortalece su posición dentro del sistema político, ya que le entrega la posibilidad de desplegar los recursos que posee como jefe del Ejecutivo para favorecer su permanencia en el cargo. Ello, además de proveer incentivos a un mayor personalismo en la política y a la corrupción (Negretto, 2009: 51). 
Uruguay en 1996, Chile en 2005, Paraguay en 2002), y en otros casos se ha contemplado directamente la posibilidad de su destitución a través del voto de la mayoría parlamentaria (por ejemplo, Colombia en 1991, Argentina en 1994). A su vez, hay también una tendencia a "introducir mayores límites y controles en materia de nombramiento de los presidentes" (Negretto, 2009: 47), que incluyen la democratización de las unidades locales, o la restricción del poder presidencial en materia de nombramiento de jueces.

Entre las reformas que destacan por el objetivo, al menos según lo que declararon quienes las acordaron, de "atenuar el sistema presidencial", se encuentra la introducción de la figura de "Jefe de Gabinete de Ministros" en el sistema institucional argentino. De acuerdo a la reforma constitucional de 1994, como se explica más adelante, éste sería un primus inter pares del gabinete, con un status institucional superior a los demás ministros, y cuya permanencia en el cargo depende del presidente y del congreso. De esta manera, esta reforma pretendía "atenuar" el poder presidencial por la vía de desconcentrar el poder al interior del Ejecutivo y de introducir "un elemento parlamentario" en el sistema presidencialista argentino (De Riz y Sabsay, 1998: 225) ${ }^{3}$, el que consistía en hacer dependiente del Congreso la permanencia en el cargo de un ministro con importantes atribuciones ${ }^{4}$. Así, el objetivo declarado fue introducir un instrumento que contribuyera a superar la rigidez del sistema presidencial por la vía de permitir al congreso destituir a un importante funcionario del Ejecutivo, limitar el poder del presidente y generar a la vez incentivos para un entendimiento (o compromiso) entre esos dos poderes para los casos de conflicto profundo entre ellos.

Éste es un muy interesante caso de estudio, ya que, transcurridos casi quince años desde la entrada en vigencia de la institución Jefatura de Gabinete de Ministros (JGM), existe experiencia suficiente para analizar en profundidad si los objetivos declarados de la reforma han sido efectivamente realizados. Considerando esto, y que el cumplimiento de

${ }^{3}$ A este respecto destaca el caso de Perú, donde desde el siglo XIX existe la figura del "Presidente del Consejo de Ministros". Aun cuando presenta diferencias importantes con el caso argentino en materia de designación y funciones, esta figura aparece formalmente también como mecanismo de atenuación del poder presidencial (a través de introducir mecanismos del parlamentarismo) y se le asigna un rol de cierta preeminencia respecto de los demás ministros (Roncagliolo, 1991; Sagües, 2006).

${ }^{4}$ Esto es muy relevante, pues en todo sistema presidencial "puro" sólo el presidente puede destituir a miembros del gabinete. De ahí que se hable de introducir un "elemento parlamentarista" al sistema político argentino (Mainwaring y Shugart, 1994). 
los objetivos de una reforma (y por tanto los efectos que puede tener sobre el funcionamiento del presidencialismo) depende tanto del diseño institucional (que puede ser ambiguo o no ser consistente con lo que formalmente se plantea como objetivo de una reforma), como de variables no institucionales, como el comportamiento de los actores frente a ella (con sus acciones u omisiones ellos pueden contribuir a realizar los objetivos buscados o bien simplemente neutralizarlos), este estudio adopta un enfoque integrado. Éste consiste en abordar el estudio de la experiencia de la JGM entre 1995 y 2007 en dos aspectos principales. El primero es analizar su marco jurídico para efecto de determinar si la introducción de la JGM implicó, al menos en lo formal-institucional, una modificación sustantiva al presidencialismo argentino, y si constituyó un mecanismo de "atenuación" del presidencialismo en los términos señalados. El segundo es examinar el proceso de asentamiento institucional de la JGM y el rol que sus titulares han jugado dentro de sus respectivos gobiernos y, por extensión, el papel real que ha jugado la JGM dentro del sistema político argentino.

Este enfoque permitirá evaluar si el diseño institucional de la JGM es adecuado a los objetivos (declarados) que motivaron su creación, así como si en los hechos el Ejecutivo asignó a la institución y su titular la importancia política que las reglas formales le asignan. Además, contribuirá a determinar si este mecanismo ayuda a superar los obstáculos que el presidencialismo aparentemente impone al funcionamiento democrático. De la misma manera, las conclusiones sobre la experiencia de la JGM serán de utilidad para quienes promuevan cambios institucionales de esta naturaleza. Este ejercicio es también útil para el caso de Chile, donde la reforma al presidencialismo fue muy debatida a principios de la década de 1990 (Godoy, 1992), a la vez que actualmente en el Congreso se discuten algunas iniciativas a ese respecto 5 .

El artículo argumenta lo siguiente: a) el diseño institucional de la JGM no es coherente con el objetivo declarado por los autores de la reforma de 1994 de atenuar el sistema presidencial, además de ser un débil mecanismo de control por parte del parlamento. Más bien, su diseño apunta a (eventualmente) desconcentrar el poder presidencial y a que la

${ }^{5}$ En abril de 2008 la Cámara de Diputados constituyó una comisión encargada de estudiar el sistema político chileno. En noviembre de 2009 aprobó un informe en que dijo era necesario estudiar "reformas necesarias para crear la figura de un primer ministro que requiera el voto de confianza del Parlamento"(www.camara.cl/prensa/noticias_detalle.aspx?prmid=36348 ). 
JGM ejerza una función de coordinación y de liderazgo dentro del gabinete, con su titular como un superior "político" de los demás ministros. b) En los hechos, la JGM no se ha consolidado en esa función, dada la continua modificación de su estructura y funciones y el diferente rol político que han tenido quienes han sido designados al frente de la JGM. En ese sentido, tampoco aporta necesariamente a una mejor dirección del gobierno. Y c), la JGM no ha sido un mecanismo útil de solución para los problemas de la rigidez del presidencialismo en casos de crisis, precisamente porque no está adecuadamente diseñada para ello.

El artículo se organiza de la siguiente manera. Primero se examina la discusión sobre presidencialismo y la literatura sobre la JGM. Luego se describe el proceso de creación de la JGM y se analiza su diseño institucional. A continuación se estudia la evolución organizacional de la JGM y se analiza el rol de los diferentes ministros que han ocupado la JGM desde la perspectiva de la función política que se les asignó y su relación con el presidente. Enseguida se hacen observaciones sobre el papel de la JGM en cuanto institución articuladora de la relación ejecutivo-legislativo, y de su utilidad como "fusible" en casos de crisis política y por tanto como mecanismo de flexibilización del presidencialismo. Por último, se exponen las conclusiones.

\section{El estudio del presidencialismo en América Latina y su proceso de reformas}

\subsection{Algunos temas de la investigación académica sobre el funcionamiento del presidencialismo}

El núcleo de la investigación académica sobre presidencialismo latinoamericano se ha desarrollado en torno a las proposiciones de Linz, quien sostiene que el presidencialismo representa, en principio, un mayor riesgo para una democracia estable que el parlamentarismo (1990: 104). En concreto, Linz sostiene que las dificultades que impone el presidencialismo ${ }^{6}$ para el establecimiento de una democracia estable son: a)

${ }^{6}$ Conforme a Linz este sistema tiene dos características definitorias centrales: a) el presidente, jefe de gobierno, es electo por votación popular, gozando entonces de total legitimidad democrática (al igual que el parlamento) y b) la elección se hace por un período fijo de tiempo, que bajo circunstancias normales no puede ser modificado. Así, se diferencia del parlamentarismo, donde sólo el parlamento tiene legitimidad democrática (1990: 46-49). 
el conflicto latente que se deriva de que tanto el presidente como el Congreso tengan legitimidad democrática (legitimidad dual); b) la rigidez que introduce al proceso político el que los presidentes sean elegidos por un período fijo, ya que no permite "la sustitución de un líder que ha perdido la confianza de su propio partido... [ni] ...su sustitución por alguien más capacitado para lograr un compromiso con la oposición cuando la polarización alcanza una intensidad tal que amenaza con violencia y con derrocamiento ilegal" (Linz, 1990: 64); c) la lógica de "juego de suma cero" que el presidencialismo impone al proceso político, donde "los vencedores y perdedores" se definen por el período del mandato presidencial, y en una situación donde los perdedores no tienen esperanza de participar del Ejecutivo (y de sus beneficios) por todo ese lapso - lo que, a su vez, tendría el efecto de aumentar la polarización y la tensión (Linz, 1990: 53)—, y d) que genera un "estilo presidencial de la política", en el que el presidente tiende a actuar solo, desestimando a los otros poderes. De esta forma, para Linz el problema central del presidencialismo es que sus características institucionales definitorias, a diferencia de lo que ocurre en el parlamentarismo, generan incentivos que afectan negativamente la estabilidad de la democracia. Esto, porque si el partido del presidente sólo tiene una minoría en el parlamento, existirá una alta probabilidad de que se produzcan fuertes conflictos entre ambos órganos. En este contexto, y ante la inexistencia de mecanismos que permitan solucionar estas situaciones de "impasse", habrá una alta probabilidad de que esos conflictos desemboquen en un quiebre institucional ${ }^{7}$.

A partir de estos argumentos se ha generado una muy abundante literatura que evalúa distintos aspectos del funcionamiento del presidencialismo $^{8}$, entre los cuales destacan los siguientes. El primero es el de la

\footnotetext{
${ }^{7}$ Aplicando esta lógica para explicar el caso del quiebre democrático en Chile en 1973, Valenzuela (1990: 180) argumenta que "en última instancia, el quiebre se debió a la imposibilidad de resolver el dilema básico de un presidencialismo de doble minoría: un ejecutivo elegido por menos del $50 \%$ del electorado y detentando apoyo minoritario en el parlamento. Al romperse la coalición original que se estructuró en el congreso y que permitió la elección del presidente minoritario, el sistema institucional chileno no proporciona soluciones institucionales al impasse entre los poderes Ejecutivo y Legislativo".

${ }^{8}$ Dado que la discusión sobre el presidencialismo y sus virtudes y defectos no es el foco central de este artículo, sólo mencionaremos algunos temas de esa discusión. Para una revisión completa, donde por ejemplo se estudian distintos esquemas sobre relaciones ejecutivo-legislativo, véanse Nolte (2004) y Morgenstern y Nacif (2002). Asimismo, otros autores se ocupan de otros temas también muy relevantes, como la interacción entre sistema electoral y régimen presidencial (Jones, 1995), las estrategias de formación de gabinetes (Amorim, 2006), las causas de las rotativas ministeriales (Martínez, 2005).
} 
estabilidad del presidencialismo, respecto de la cual Mainwaring y Shugart sostienen que es cierto que en principio los regímenes presidenciales son más inestables que los parlamentarios, aun cuando advierten que la conclusión se debe tomar con cautela pues muchos de los intentos por establecer regímenes presidencialistas han tenido lugar en países subdesarrollados" (1994: 401; véanse también Cheibub y Limongi, 2002). Relacionado con esto, se ha analizado el conflicto entre los poderes ejecutivo y legislativo de los regímenes presidenciales en América Latina. Pérez-Liñán (2003), a partir de un estudio del período 1945-2000, sostiene que si bien el conflicto ejecutivo-legislativo (en el marco de regímenes presidenciales) está presente durante todo ese lapso, a partir de la década de 1990 la tasa de quiebres democráticos producto de conflicto entre poderes ha descendido muy notablemente.

En este contexto, el estudio de los factores que afectan la estabilidad del presidencialismo ha sido objeto de intensa investigación, argumentando Shugart y Carey que los sistemas problemáticos son aquellos en los que el presidente detenta mayores poderes (formales) y en que presidente y parlamento comparten la autoridad sobre el gabinete (1992: 148-166; véanse también Mainwaring y Shugart 1994; Payne 2007). Sin embargo, Cheibub (2007), a partir de un examen estadístico de la correlación entre algunas dimensiones del poder presidencial (poderes de veto $\mathrm{y}$ facultades presupuestarias) y estabilidad, señala que no hay una correlación entre mayores poderes presidenciales e inestabilidad.

Desde otra perspectiva, Mainwaring sostiene que el problema no está en el presidencialismo per se (su forma institucional), sino en su combinación con el multipartidismo, que hace aumentar la probabilidad de un bloqueo (deadlock) entre ejecutivo y legislativo, que inmovilice al sistema político. Ello, pues la mayor fragmentación del sistema dificulta que un presidente goce de apoyo mayoritario en el parlamento y abre más espacios a la polarización ideológica (1993: 214-215). Chasquetti refuta parcialmente el argumento, ya que la clave es que los "presidentes sean capaces de conformar coaliciones de gobierno de carácter mayoritario" (2001: 346), lo que no es imposible pues existen mecanismos que sirven de base para ello (por ejemplo, las facultades de realizar nombramientos tanto en el gabinete como en las instituciones estatales). Cheibub y Limongi analizan la formación de coaliciones en el presidencialismo y afirman que este sistema necesariamente no las desincentiva, y que a medida que hay mayor distancia en materia de preferencias de 
política pública (policy space) entre el partido del presidente y los de oposición, mayor es el incentivo para formar coaliciones. De hecho, agregan, en el presidencialismo las coaliciones se forman casi la mitad del tiempo en que no hay un partido mayoritario (2002: 166; véase también Foweraker, 1998). Desde otra perspectiva, el examen que realiza Thibaut (1996) sobre la formación de coaliciones en Brasil, Argentina, Chile y Uruguay entre 1983 y 1996, confirma que el presidencialismo es compatible con la formación de coaliciones y con esquemas de cooperación entre presidente y parlamento.

Los enormes avances en el conocimiento del presidencialismo en relación a los temas indicados, y muchos otros no reseñados, son ciertamente de gran utilidad. Sin embargo, en buena parte de la literatura los énfasis investigativos están más en el rol de variables institucionales ${ }^{9} \mathrm{y}$ menos en los aspectos fácticos del funcionamiento de las instituciones. Así, salvo excepciones sobre estudios de casos específicos (por ejemplo, Huneeus, 2005; Mustapic, 2002; Siavelis, 2000), aspectos como la forma en que se estructura la relación presidente-gabinete, entre el presidente y los partidos, o como se utilizan recursos de poder no institucionales (como el rol de los staff presidenciales) tienen un escaso desarrollo ${ }^{10}$. Por tanto, este trabajo se inserta en la línea de aquellos en que se exploran no sólo las variables institucionales, sino que también aspectos fácticos del funcionamiento del sistema presidencial.

2.2. El estudio del funcionamiento de los mecanismos de atenuación del presidencialismo

A diferencia de lo que ocurre con el estudio de los problemas centrales del presidencialismo planteados por Linz, en el plano concreto de las reformas que se han introducido en diversos países para darle una

${ }^{9}$ Esta visión institucionalista-formal es predominante también en los estudios sobre medición del poder presidencial, como ocurre en la obra de Shugart y Carey (1992), quienes proponen medirlo a partir de las atribuciones formales de los presidentes, legislativas y no legislativas. Este criterio es el que mantienen otros esquemas, pero que se diferencian a partir del número de variables que consideran (por ejemplo, Mainwaring y Shugart, 1994; Metcalf, 2000).

${ }^{10}$ Me refiero aquí principalmente a la literatura sobre América Latina. Distinto es lo que ocurre en el estudio del presidencialismo de Estados Unidos, en cuyo marco se ha examinado, por ejemplo, la institucionalización y crecimiento del staff presidencial, elemento esencial del fortalecimiento de la presidencia desde la década de 1940 (Dickinson, 2005; Dickinson y Lebo, 2002; Kessell, 1984). 
cara "más parlamentaria" al sistema presidencial, como es el caso de la JGM o de la presidencia del Consejo de Ministros en el Perú, o respecto de las que aumentan el poder del control del Congreso sobre el Ejecutivo, no existe una amplia literatura que las analice desde una perspectiva integrada, como aquí se propone. De hecho, en el caso peruano son pocos los trabajos que analizan en profundidad esta institución desde una perspectiva más allá de lo formal. Esto, sin perjuicio del valor que ellos tienen y de que en general coinciden en que a pesar de la existencia de esta "institución parlamentaria" en el sistema político peruano, en los hechos éste sigue siendo fuertemente presidencial (Eguiguren, 2004; Sagües, 2006). Asimismo, existen pocos trabajos sobre las reformas que buscan aumentar el poder de control del parlamento. En el caso de Chile, por ejemplo, y lo que se explica fundamentalmente por lo reciente de la reforma de 2005, no existen trabajos que analicen el funcionamiento práctico de estos instrumentos, como la interpelación parlamentaria. Ello, con la excepción de aquellos que los estudian desde una perspectiva formal (Carmona, 2006), o hacen una investigación fundamentalmente exploratoria (Berríos y Gamboa, 2006).

En el caso argentino, en particular respecto a cómo la introducción de la JGM afecta el funcionamiento del sistema presidencial o las dificultades que ha tenido para establecerse como institución, se observa la misma falencia. En efecto, los pocos análisis sobre la institución lo han hecho estudiando sus orígenes y estatuto constitucional (Barraza y Schafrik, 1999; Bidart, 1995; De Riz y Sabsay, 1998; Quiroga, 2005), su evolución organizacional (Novaro, 2001), o bien otros aspectos como el rol de los jefes de gabinete en el marco de crisis políticas (Serrafero, 2003). De esta forma, y a pesar del aporte que estos trabajos realizan, es claro que una evaluación de la JGM como eventual mecanismo para desconcentrar el poder presidencial y ser vía de solución para crisis entre los poderes legislativo y ejecutivo, y de su rol efectivo dentro del sistema institucional, se hace absolutamente necesaria. Y esto, como se dijo, realizando un análisis que abarque tanto su diseño institucional como la experiencia práctica de su funcionamiento.

En este marco, en las páginas siguientes se intenta avanzar en la dirección indicada, analizando el proceso de establecimiento de la JGM y su rol efectivo dentro del sistema presidencial argentino, lo cual abarca incluso aspectos que van más allá de su eventual aporte como mecanismo de atenuación del presidencialismo. Para ello, se utiliza un 
esquema que integra tanto aspectos institucionales como fácticos, en particular la posición efectiva que se le ha asignado dentro de cada gobierno a la JGM.

\section{La Jefatura de Gabinete de Ministros en Argentina desde 1995}

\subsection{Orígenes: la reforma constitucional de 1994}

El debate sobre la necesidad de atenuar el sistema "hiperpresidencialista" argentino (Nino, 1988) resurgió con fuerza a partir de $1983^{11}$. Un punto central en esta materia fue la proposición de incluir la figura de un "jefe de gabinete" hecha por el "Consejo para la Consolidación para la Democracia”, que presidió el destacado constitucionalista Carlos Nino. Si bien en esta instancia no hubo consenso para establecer un sistema semipresidencial como el francés, ya que no podía constituirse un "ejecutivo bicéfalo" (Barraza y Schafrik, 1999: 132-133), se propuso, por un lado, desconcentrar las funciones del presidente, entregando a otra institución (jefe de gabinete) las tareas administrativas y dejando al primero la conducción estratégica del gobierno. Por el otro, el jefe de gabinete se constituiría en vía de comunicación entre el Ejecutivo y el Congreso, y podría ser destituido tanto por el presidente como por el Congreso (ibídem, 137). Aun cuando los proyectos reformistas impulsados por Alfonsín no pudieron concretarse, la discusión se retomó en la década de 1990, cuando por iniciativa del presidente Menem (1989-1999), que buscaba reformar la Constitución para poder optar a la reelección (Negretto, 2001: 142), se introdujo una reforma constitucional por la cual, entre otras cosas, se creó la institución de la "Jefatura de Gabinete de Ministros".

La reforma constitucional ${ }^{12}$ fue negociada entre el líder de la Unión Cívica Radical (UCR), Raúl Alfonsín, y Menem, materializándose un primer acuerdo en noviembre de 1993, conocido como "Pacto de Olivos". En éste, ellos declararon su acuerdo en impulsar una reforma constitucional que consolidara el sistema democrático y perfeccionara

${ }^{11}$ El debate sobre esta materia es más antiguo, teniendo antecedentes en los inicios del siglo XX. Para el efecto, véanse Serrafero (2003: 249-252) y Negretto (2001: 135-139).

12 Existe amplia literatura a este respecto, entre otros, véanse Quiroga (2005), Negretto (2001), García Lema (1994), De Riz y Sabsay (1998). Para la visión de uno de sus actores, véase Alfonsín (1996: 263-335). 
el equilibrio de poderes. Esto se haría a través de distintas reformas, entre las que se incluía "la atenuación del sistema presidencialista por medio de la incorporación de un Jefe de Gabinete o Ministro coordinador, con responsabilidad frente al Presidente y al Congreso" (San Martino, 1996: 1214). Un segundo acuerdo, conocido como "Pacto de la Rosada", de diciembre de 1993, estableció las bases específicas de la reforma constitucional, dividiendo su contenido en dos partes. En la primera, denominada "Núcleo de Coincidencias Básicas", se determinaron un conjunto de normas bajo el título de "Atenuación del sistema presidencialista" y que establecían el marco jurídico del "Jefe de Gabinete de Ministros". Otras disposiciones introdujeron la posibilidad de reelección presidencial, la reducción del mandato presidencial a cuatro años, el cambio del sistema de elección de senadores y del presidente, entre otras. Se acordó que el "Núcleo de Coincidencias" podría ser analizado por la Convención Constituyente que debía elegirse, pero con la limitación de que no podía modificar su contenido, pudiendo sólo aprobar o rechazar lo acordado (Jones, 1997; Quiroga, 2005). En la segunda, se listaron diversos temas que podrían ser objeto de reforma. En abril de 1994 se eligió la Convención, la que aprobó el "Núcleo..." en la forma acordada (San Martino, 1996: 1214 y sgtes).

Visto en términos globales, el acuerdo Menem-Alfonsín tuvo un carácter mixto, pues la posibilidad de reelegir al presidente en ejercicio no implica atenuar el presidencialismo, sino eventualmente fortalecerlo. Asimismo, la creación de la JGM, declarada como el principal mecanismo de atenuación del presidencialismo argentino, fue en definitiva una concesión que Menem hizo a la UCR para lograr se aprobara la reelección presidencial, que era su principal objetivo.

\subsection{El marco institucional}

Los artículos 99 a 101 de la Constitución reformada en 1994 establecen el marco jurídico fundamental de la JGM. En cuanto al nombramiento y destitución de su titular, el artículo 99 dispone que el titular de la JGM es designado y removido libremente por el presidente, al igual que los demás ministros. A ello, el artículo 101 agrega que puede ser removido por el Congreso, para lo cual se requiere el voto de la mayoría absoluta de los miembros de cada una de sus cámaras. 
De acuerdo a Serrafero (2003), se distinguen cuatro tipos de atribuciones de la JGM: políticas, administrativas, económicas y relacionadas con el Congreso. Ellas se resumen en el Cuadro $\mathrm{N}^{\circ} 1$.

Administrativas

Relación Ejecutivo-Congreso
a) Ejercer la administración general del país;
b) Efectuar los nombramientos de los empleados de la admi- nistración, excepto los que corresponden al presidente;
c) Expedir actos y reglamentos que sean necesarios para ejer- cer sus facultades y las que le delegue el presidente, y,
d) Ejercer las atribuciones que le delegue el presidente y en acuerdo con el gabinete resolver sobre las materias que indi- que el ejecutivo (art. 100)

a) Ir a las sesiones del Congreso y participar en sus debates (sin derecho a voto);

b) Producir los informes y explicaciones que cualquier cámara solicite al Ejecutivo;

c) Presentar, antes del inicio de las sesiones ordinarias del Congreso y junto a los demás ministros, una memoria sobre el estado de la Nación;

d) Concurrir, al menos una vez al mes, al Congreso (alternativamente a cada cámara) para informar de la marcha del gobierno, y,

e) Refrendar los decretos reglamentarios de las leyes y los mensajes del presidente que promuevan una iniciativa legislativa.

Económicas

a) Enviar al Congreso los proyectos de ley de ministerios y de presupuesto, previa aprobación del presidente, $y$,

b) Hacer recaudar las rentas de la nación y ejecutar la ley de presupuestos.

Políticas

a) Convocar y coordinar las reuniones de gabinete y presidirlas en ausencia del presidente;

b) Refrendar (por sí solo) decretos que ejercen facultades delegadas, y en conjunto con los demás ministros los decretos de necesidad y urgencia y los decretos que promulguen parcialmente las leyes (en todos los casos esos decretos deben ser luego conocidos por una Comisión Bicameral permanente).

Visto el estatuto de la JGM, es necesario analizarlo en términos de la posición que se le asigna dentro del sistema institucional y de si constituye, como sostuvieron los padres de la reforma de 1994, un mecanismo de atenuación del presidencialismo. 
Una primera observación es que la inserción de la JGM no alteró la naturaleza del sistema político argentino. Si bien éste no fue un objetivo expreso de la reforma, durante su discusión estuvo presente la idea que a través de la JGM se buscaba darle al sistema una cierta "fachada parlamentarista" (entrevista Mora y Araujo, 2008). Con todo, es claro que ella no fue un paso hacia otra forma de gobierno, por cuanto la misma Constitución establece que el presidente es "jefe supremo de la Nación, jefe del gobierno y responsable político de la administración general del país" (Art. 99). De esta manera, la reforma mantuvo los elementos centrales de un sistema presidencial, como la existencia de un presidente electo por votación popular, y a la vez de un parlamento que también es electo democráticamente, y de cuya voluntad no depende la estabilidad del gobierno, ni de su jefe (el presidente es electo por un período fijo). Consecuentemente, la posición institucional del titular de la JGM es muy distinta a la de un primer ministro en un sistema parlamentario, en el cual sólo el parlamento es electo democráticamente, y de cuya voluntad depende la permanencia en el cargo del primer ministro (jefe de gobierno) y del resto del gabinete ${ }^{13}$. Asimismo, el diseño tampoco se asemeja a un sistema semipresidencial, pues en éste existe una estructura dual de poder. Esto, porque aquel se caracteriza, por un lado, por la existencia de un presidente electo por votación popular y, por el otro, de un "primer ministro" que concentra el poder ejecutivo (lo que no ocurre en Argentina para el caso del jefe de gabinete) y que permanece en el cargo mientras no sea destituido por el parlamento (Sartori, 1994: 137) ${ }^{14}$. Así, el único punto de contacto, en lo formal, entre el sistema argentino y el parlamentarismo y el semipresidencialismo (aparte de con este último comparte el hecho de que hay un presidente electo democráticamente) es que existe la posibilidad de que el jefe de gabinete sea destituido por el parlamento, aun cuando el presidente se oponga a ello. Con todo, la distancia respecto de ambos sigue siendo

\footnotetext{
${ }^{13}$ Para un examen de los elementos que definen el parlamentarismo, las diferentes formas organizativas que puede adquirir y de las atribuciones que normalmente posee un primer ministro, véase Pasquino (2007).

${ }^{14}$ Existe un intenso debate, no definido aún, en torno a si se debe incluir dentro de las características de un sistema semipresidencial el que el presidente tenga amplios poderes ejecutivos (por ejemplo, veto, iniciativa legislativa, etc.). No obstante, existe pleno acuerdo en que las dos características citadas son parte esencial de estos sistemas. Para este debate, véase Duverger (1980) y Elgie (2008).
} 
muy grande, pues en el caso argentino el titular de la JGM no es el jefe de gobierno, sino que lo es el presidente de la nación ${ }^{15}$.

Visto lo anterior, lo segundo a subrayar es que lo que realmente estableció la reforma fue la "desconcentración" del poder ejecutivo por la vía de la delegación (Serrafero, 2003: 252), pero sin que la JGM sea autónoma. Esto, por un lado, porque en caso de desacuerdo con las acciones de su jefe de gabinete el presidente puede destituirlo sin necesidad del acuerdo de nadie. Por el otro, porque varias de las atribuciones que ejerce o puede ejercer el jefe de gabinete lo son por delegación del presidente o en último término está sometido a él. Prueba de ello es que su facultad de "ejercer la administración general del país" está limitada por ser el presidente el jefe de gobierno y responsable político de la administración: es decir, al jefe de gabinete le corresponde el ejercicio de la administración, mientras al presidente la "titularidad de la misma" (Quiroga 2005: 204). El jefe de gabinete carece entonces de autonomía, ya que el presidente posee todos los dispositivos para hacer prevalecer su voluntad sobre el titular de la JGM en caso de que exista una discrepancia entre ellos.

En tercer lugar, cabe señalar que el jefe de gabinete tiene una posición institucional distinta a la de los demás ministros. Primero, porque es el único que tiene una doble dependencia respecto de su permanencia en el cargo (del Ejecutivo y del parlamento). Segundo, porque tiene una posición (formal) de preeminencia. Esto, no sólo por el nombre que

${ }^{15}$ En el caso del "Presidente del Consejo de Ministros" (PCM) en Perú, la figura es distinta. El artículo 121 de la Constitución de 1993 dispone que éste es designado por el presidente, quien además puede removerlo del cargo a voluntad. En relación a los demás ministros, el presidente resuelve respecto de su nombramiento "a propuesta y con acuerdo" del PCM (art. 122). Lo mismo respecto a su remoción.

Una diferencia sustantiva con la JGM está en que, dentro de los 30 días posteriores a su designación (art. 130), el PCM debe presentar al Congreso los principales lineamientos del plan de gobierno, y plantear al efecto una "cuestión de confianza" para ser aprobada o rechazada por el parlamento. A lo anterior, se agrega que en cualquier tiempo el Congreso tiene la facultad de censurar al Consejo de Ministros o a cualquier ministro, exigiéndose para la aprobación de un voto de censura de la mayoría de los miembros del parlamento. Si la censura prospera, el Consejo o el ministro censurados deben renunciar y el presidente aceptar la dimisión.

Con todo, y en otra diferencia importante, también se establece que en caso de que el Congreso censura o niega la confianza a dos consejos, el presidente puede disolver el parlamento (art. 134). Con ello el sistema peruano se asemeja más a un sistema semipresidencial, aun cuando el presidente (electo por un período fijo) es quien sigue teniendo las principales funciones ejecutivas, siendo el verdadero jefe de gobierno (véase Sagües, 2006). 
lleva, sino también, por ejemplo, por sus atribuciones sobre realización de reuniones de gabinete, las relativas al presupuesto o las de refrendar actos del Ejecutivo en materia de iniciativa legislativa. De esta manera, según Barraza y Schafrik, el jefe de gabinete se impone como "un superior jerárquico de los ministros secretarios" (1999: 122).

En consecuencia, la conclusión en esta materia es que el jefe de gabinete debe entenderse, al menos en un principio, no como una figura de atenuación del sistema presidencial conforme lo señalaron Menem y Alfonsín, sino como un (formalmente) primus inter pares dentro del gabinete y coordinador de la actividad gubernamental, tanto respecto a la relación interministerial como a la interlocución con el Congreso.

No obstante la importancia del examen de las normas que regulan la JGM, una evaluación más completa de su rol exige examinar otros aspectos de su actividad, como son la evolución de su estructura institucional y la posición de la JGM dentro de cada gobierno. Esto es útil y necesario, pues permitirá apreciar cómo la JGM efectivamente mantiene y consolida el control de las diferentes áreas, y cuál es la experiencia de los titulares de la JGM como efectivos (o no) "superiores jerárquicos" de los demás ministros, dentro de lo cual un aspecto esencial es analizar quiénes ocupan el cargo y la función política que le asigna el presidente.

\subsection{La estructura institucional: variación de competencias}

La JGM entró en funciones en julio de 1995, fijándose en ese momento su primera estructura institucional, la que posteriormente sufrió diversas modificaciones. En ese marco, lo primero que resalta es que en 13 años de funcionamiento aún no se ha logrado establecer y consolidar una estructura institucional. Esto, por cuanto si bien hay áreas que siempre han estado bajo su dominio, hay otras que se han agregado posteriormente y otras que han sido traspasadas a otros ministerios. Con todo, cabe subrayar que ello no es un problema exclusivo de la JGM, ya que en este período otros ministerios también han sufrido cambios importantes a través de fusiones con otros ministerios, o traspaso de funciones, o creación de otras instituciones. En segundo lugar, destaca el que en los hechos la JGM ha tenido dificultades para ejercer efectivamente las atribuciones que se le asignan, principalmente a causa de disputas con otros ministerios o por decisiones presidenciales que, de 
hecho, traspasan a otras instituciones las atribuciones que formalmente le corresponden a la JGM.

Respecto del primer punto, una breve revisión de la evolución de su estructura interna confirma lo señalado ${ }^{16}$. En efecto, en 1995 se definió una estructura que contemplaba 6 secretarías — planeamiento, coordinación de gabinete, coordinación parlamentaria, asuntos fiscales, administración y control estratégico- cada una con 2 subsecretarías. La primera tenía como tarea principal el diseño de políticas de administración, en especial lo referido a la transformación estructural del Estado, mientras la segunda conducía las relaciones con los ministerios y colaboraba en la elaboración de los informes a los ministerios. Esto último lo realizaba en conjunto con la de coordinación parlamentaria, la que también hacía el seguimiento legislativo y colaboraba en tareas relacionadas con la delegación de facultades y reglamentación de leyes. Por último, la de asuntos fiscales ejercía las atribuciones en materia presupuestarias y de recaudación (Novaro, 2001). Esta estructura inicial, con evidentes problemas de superposición de funciones, fue modificada en sucesivas oportunidades entre 1996 y 1997, quedando conformada por cuatro secretarías, que perduraron hasta 1999: control estratégico (encargada de seguimiento de políticas), equidad fiscal (competente en materias presupuestarias y de recaudación y que por un tiempo había estado bajo el alero de la de coordinación), relaciones parlamentarias e institucionales (que conducía la relación con el parlamento, y que absorbió las que antes tenía la secretaría de coordinación de gabinete) y la de función pública (encargada de proponer reformas a la administración) (Novaro, 2001).

Durante el gobierno de De la Rúa (1999-2001) hubo nuevas modificaciones, quedando sólo una secretaría (coordinación general) y cuatro subsecretarías que ejercían básicamente las funciones de las que antes eran secretarías. Esta organización cambió nuevamente durante el gobierno de Duhalde (2002-2003), mientras que con la llegada de Kirchner a la presidencia en 2003 se definió una nueva estructura que se mantuvo hasta 2007. Lo llamativo de esa nueva estructura es que, aparte de mantener una secretaría de gabinete y relaciones parlamentarias y las instancias encargadas de las tareas vinculadas al presupuesto y gestión pública (aun cuando estas dos últimas como subsecretarías), se agre-

\footnotetext{
${ }^{16}$ Agradezco a Paula Orsini la información sobre la evolución organizacional de
} la JGM. 
garon tres secretarías que antes no estaban en la órbita del ministerio: Deportes, medios de comunicación y ambiente y desarrollo sustentable. Es decir, con esto se ampliaron las competencias de la JGM a tres áreas antes ajenas a ella. Sin embargo, en diciembre de 2007 hubo nuevos cambios, retirándose deportes como área de competencia de la JGM.

Lo visto demuestra lo dicho en términos de que si bien la JGM desde sus inicios ha centrado su actividad en ciertas áreas (presupuestaria, relaciones con el Congreso, y coordinación interministerial), las estructuras específicas encargadas han sufrido permanentes modificaciones, ya que muchas han cambiado alternativamente de rango, siendo secretarías o subsecretarías, o bien unidades dentro de ellas. A ello se agrega que su campo de atribuciones ha sido "variable", en términos de que hay ámbitos que se han agregado y/o quitado a la JGM a lo largo del tiempo, como es el caso de las secretarías mencionadas (por ejemplo, Deporte) y programas específicos, como el Fondo Fiduciario Federal de Infraestructura Regional (entrevista con Orsini, 2008; Novaro, 2001).

En cuanto al segundo punto, ha existido una dificultad permanente de la JGM de ejercer efectivamente las atribuciones que se le encomiendan. Un caso ejemplar es el presupuestario, materia sobre la que ha existido una permanente disputa entre la JGM y el Ministerio de Economía. Durante el gobierno de Menem y mientras fue ministro de Economía Cavallo (1991-1996), hubo una fuerte resistencia de éste para que el tema quedara bajo la dirección de la JGM. Esto se modificó luego con la salida de Cavallo del cargo, mejorándose la posición relativa de la JGM, aun cuando esto no significó que ésta adquiriera una posición dominante en materia presupuestaria, ya que a partir de ahí la Secretaría de Hacienda (del Ministerio de Economía) siguió concentrando los aspectos técnicos y la JGM se quedó con el manejo de la política de "negociación y resolución de conflictos" en esta materia (Novaro, 2001). Durante la gestión de De la Rúa persistieron los roces, los que se manifestaron en una fuerte disputa entre el ministro de Economía José Luis Machinea y el jefe de gabinete, Rodolfo Terragno. A partir de 2003, esta situación tuvo un cambio importante al establecerse un nuevo marco institucional, que delineó en forma más clara el rol que a cada institución correspondía. De acuerdo al subsecretario de evaluación del presupuesto, Norberto Perotti, en 2003 se configuró (con el apoyo del BID) una nueva estructura dentro de la JGM para tratar el tema 
presupuestario, que era muy superior a la anterior, tanto en términos de recursos como organizacionales. A la vez, se delineó una repartición de tareas entre el Ministerio de Economía y la JGM, siendo el Ministerio de Hacienda, a través de la Secretaría de Hacienda, el encargado de elaborar las partidas presupuestarias ${ }^{17}$, mientras la JGM dirige la coordinación política para la elaboración del presupuesto, además de hacer el seguimiento de la ejecución presupuestaria y la evaluación de cumplimiento de metas (entrevista con Perotti, 2008).

Otro ejemplo de debilidad de la JGM se da en materia de recaudación, donde la instancia encargada de ejecutar la política tributaria, la Administración Federal de Ingresos Públicos (AFIP), tampoco está bajo la dependencia de la $\mathrm{JGM}^{18}$, no obstante sus funciones en materia de recaudación de ingreso (véase Cuadro $\mathrm{N}^{\circ} 1$ ). Al respecto, ésta sólo participa en la definición del Plan de Acción que la AFIP desarrolla cada año para determinar como va a cumplir las metas de recaudación (entrevista con Perotti, 2008).

\subsection{Los jefes de gabinete en tres gobiernos: Menem, De la Rúa y} Kirchner

Antes de examinar el rol de los distintos ministros que han estado al mando de la JGM, cabe subrayar como cuestión preliminar que desde julio de 1995 hasta julio de 2008 siete personas desempeñaron el cargo. De ellas, sólo dos lo hicieron por más de dos años (véase Cuadro $\mathrm{N}^{\circ} 2$ ). Esta alta rotativa ha estado influida por la difícil coyuntura del gobierno de De la Rúa y la inestabilidad derivada de la crisis seguida luego de su caída, de forma tal que entre el principio del gobierno de De la Rúa (diciembre 1999) y fines del de Duhalde (abril 2003) hubo cuatro ministros. En cambio, en los gobiernos de Menem y Kirchner la rotación es menor, ya que en el caso del primero hubo dos ministros, mientras en el segundo sólo uno.

${ }^{17}$ Esto se justifica por la mayor competencia técnica que tiene la Secretaría de Hacienda, que cuenta con 200 funcionarios, mientras que en la JGM sólo 35 personas laboran en materias presupuestarias (entrevista con Perotti, 2008).

${ }^{18}$ La AFIP es definida como una "entidad autárquica en el orden administrativo" (decreto 618/97), pero que está bajo la superintendencia general y control de legalidad del Ministerio de Economía. De ella dependen las dos principales instituciones encargadas de la recaudación: la Dirección de Ingreso Públicos (DGI) y la Dirección General de Aduanas (DGA) 


\begin{tabular}{llllll}
\hline Nombre & Partido & Profesión & Nombramiento & Salida & Presidente \\
\hline Eduardo Bauzá & PJ & Abogado & $8-6-1995$ & $28-3-1996$ & Menem \\
Jorge Rodríguez & PJ & Agrónomo & $28-3-1996$ & $10-12-1999$ & Menem \\
Rodolfo Terragno & UCR & Abogado & $10-12-1999$ & $5-10-2000$ & De la Rúa \\
Chrystian Colombo & Ind. & Economista & $5-10-2000$ & $20-12-2001$ & De la Rúa \\
Jorge Capitanich & PJ & Contador & $3-1-2002$ & $2-05-2002$ & Duhalde \\
Alfredo Atanasof & PJ & Dir. Sindical & $3-05-2002$ & $22-5-2003$ & Duhalde \\
Alberto Fernández & PJ & Abogado & $23-5-2003$ & $23-7-2008$ & Kirchner \\
& & & & & Fernández \\
Sergio Massa & PJ & n/d & $23-7-2008$ & --------- & Fernández \\
\hline
\end{tabular}

Subrayar esta alta rotativa ministerial es importante pues ella contribuye a la dificultad que ha tenido la JGM para encontrar un lugar definido dentro del sistema, ya que los constantes cambios de ministro indudablemente impiden la continuidad del proceso de asentamiento de una institución. Sin embargo, esto dice poco acerca del rol específico que cada ministro jugó dentro de su gobierno, de si durante su gestión se constituyeron como efectivos primus inter pares respecto de los demás ministros y si en los hechos, y a pesar de su diseño institucional, la JGM ha significado un cambio en el modo de funcionar del presidencialismo argentino. Por esto, considerar qué personas son las designadas para dirigir el ministerio y examinar su rol específico dentro del gabinete es fundamental para entender la posición que en cada gobierno se le asigna a la JGM y a partir de ello evaluar si cumple las funciones que en lo formal le corresponden. Esto es lo que se realiza en las siguientes páginas, lo que sin embargo se restringe sólo a los ministros de los gobiernos de Menem, De la Rúa y Kirchner, omitiéndose el análisis de los ministros de Duhalde, ya que su presidencia tuvo un carácter transitorio.

\section{a) La JGM con Menem (1995-1999)}

La JGM entró en funciones en 1995, luego de la reelección de Menem como presidente. Por su parte, Domingo Cavallo seguía al frente del Ministerio de Economía, desde donde, gracias a su exitosa gestión, había logrado consolidar una fuerte posición política al interior del gabinete. Sin embargo, hacia 1995 su relación con Menem mostraba signos de deterioro, además de existir otros ministros que no aceptaban 
el liderazgo de Cavallo e intentaban disputar su poder buscando una mayor influencia sobre Menem. De esta forma, en el gabinete se configuraron dos "alas", una "técnica" y otra "política", que en adelante comenzaron una lucha por obtener mayores cuotas de poder. Ante este escenario, Menem tomó una posición más distante de Cavallo y jugó más un rol de árbitro buscando mantener el equilibrio entre los grupos (entrevista con Acuña, 2008; Novaro, 2001).

En ese marco, el primer titular de la JGM fue Eduardo Bauzá, un abogado de larga militancia en el Partido Justicialista (PJ). Cuando Menem fue gobernador de La Rioja, Bauzá fue secretario de Desarrollo (1973-1976), para luego durante su gobierno ocupar distintos cargos antes de llegar a la JGM: ministro del Interior (1989-1990) y de Salud (1993-1995), además de haber sido secretario de la presidencia. A Bauzá le correspondió crear el nuevo ministerio y desde ahí servir de contrapeso al poder de Cavallo en el gabinete como líder del ala "política", donde cumplió una efectiva labor, convirtiéndose en el "jefe político de la administración" con el total apoyo de Menem, mientras Cavallo era el jefe económico (De Riz/Sabsay, 1998: 231). Sin embargo, su gestión fue breve. Renunció en marzo de 1996 por razones de salud, por lo cual su tarea de formación institucional quedó incompleta, además de no poder lograr consolidar un espacio claro para la JGM.

Su sucesor fue Jorge Rodríguez, diputado por el PJ entre 1989 y 1992 y ministro de Educación entre 1992 y 1996. A pesar de estos antecedentes, él no formaba parte del círculo cercano a Menem, como sí lo eran otros miembros del gabinete, en especial el ministro del Interior Carlos Corach (La Nación 29 y 31. 3. 1996). En este contexto, su nombramiento fue interpretado como una señal de debilitamiento de la JGM, no obstante se procuró mantener un marco de equilibrio entre los sectores político y económico del gobierno, que liderarían Corach y Cavallo (luego Roque Fernández), respectivamente. Así, y aun cuando en su gestión se organizó institucionalmente a la JGM, él no era una persona de la mayor confianza política del presidente, por lo que si bien ejecutaba decisiones dentro de su órbita de atribuciones, no era un líder del gabinete. Consecuentemente, durante su gestión la JGM no lideró la "administración política del gobierno", ya que esta tarea estuvo concentrada en el ministerio del Interior, encabezado por Corach, líder del "gabinete político" (entrevista con Serrafero, 2008) ${ }^{19}$.

${ }^{19}$ A ello se sumaba que Bauzá, desde su cargo de senador, seguía siendo asesor informal de Menem. 


\section{b) Los ministros de De la Rúa (1999-2001).}

Este gobierno fue el primero desde 1983 que se apoyó en una coalición de partidos, que eran la UCR (de la cual De la Rúa era el líder de su ala conservadora) y el FREPASO ${ }^{20}$, lo que generó un contexto particular para la constitución del gabinete, exigiendo a De la Rúa dar cabida a los dos partidos y las corrientes internas de la UCR. La decisión fue formar un gabinete que, además de congregar a varios economistas de primera línea, diera espacio a los distintos grupos en que se apoyaba el gobierno ${ }^{21}$. La designación como ministro de la JGM recayó en Rodolfo Terragno, ex ministro de Obras Públicas durante el gobierno de Alfonsín (1983-1989) y perteneciente al ala progresista del partido ${ }^{22}$. Así, su designación debe entenderse no como un acto por el cual De la Rúa lo encumbraba como un articulador del poder presidencial, sino como parte del equilibrio que se necesitaba para que funcionara la coalición (entrevista con Novaro, 2008).

En un contexto en que el gobierno de De la Rúa se caracterizó por un progresivo debilitamiento, lo central respecto a la gestión de Terragno fue que siempre estuvo en conflicto con otros sectores del gobierno, el que se originaba en las diferencias respecto de la política económica que debía seguirse: de un lado estaba "el sector económico" (liderado por el ministro de Economía, Machinea), y del otro los sectores del FREPASO y radicales "no-delaruistas", que se oponían a un manejo económico ortodoxo y liberal, que representaba Machinea. Desde su cargo, Terragno buscó liderar el sector "no-liberal", impulsando la creación de un "gabinete social", que buscaría generar políticas sociales distintas a las que se venían desarrollando (entrevista con Novaro, 2008). Esto provocó diversos roces entre la JGM y Economía, situación en la cual De la Rúa optó por apoyar al sector "liberal", el que consolidó su posición luego del reemplazo de Terragno en octubre de 2000. De esta manera, Terragno no se constituyó nunca en un articulador del gobierno y del gabinete, estando su gestión marcada por falta de apoyo

${ }^{20}$ Para una descripción de la constitución del FREPASO y la formación de la coalición con la UCR conocida como "Alianza por el Trabajo, la Justicia y la Educación”, véase Fernández (2007) y Leiras (2007: 137-149).

${ }^{21}$ De hecho, según Graciela Fernández, de los diez ministros sólo tres eran personas cercanas al presidente (Fernández, 2007: 161).

${ }^{22}$ De hecho, había competido por la nominación radical en contra de De la Rúa. 
presidencial, ausencia de liderazgo y una permanente tensión con Economía ${ }^{23}$.

Distinto fue el caso de su sucesor, el economista independiente Chrystian Colombo. Antes de su nombramiento fue presidente del Banco Nación, donde desarrolló una buena interlocución con los gobernadores del PJ, además de una fluida relación con De la Rúa, quien lo veía cercano a las políticas de Machinea (Terragno 2004; La Nación 6.10.2000). De esta forma, además de dar más "homogeneidad" al gabinete, su nombramiento fue una señal de querer en el cargo a una persona de mayor confianza y que esta vez sí actuaría como un coordinador. Eso fue lo que efectivamente ocurrió, pues Colombo sí jugó un rol central dentro del gabinete, con mayor participación en la ejecución de la política presupuestaria ${ }^{24}$ y como efectivo interlocutor entre el ejecutivo y la mayoría peronista en el parlamento (entrevistas con Novaro y Serrafero, ambas 2008). Esto, inclusive luego de que Domingo Cavallo retornó al gabinete. De hecho, en las horas finales del gobierno en diciembre de 2001, el ministro Colombo aparecía como su último sostén y el único con fuerza para intentar una salida alternativa a la crisis (un gobierno de coalición con la oposición). Tanto así, que en medio del descalabro no se planteó destituir a Colombo, quien "era el más sólido cuando el gobierno se licuaba aceleradamente" (Serrafero, 2003: 264).

\section{c) El gobierno de Kirchner (2003-2007)}

Alberto Fernández como titular de la JGM en el gobierno de Néstor Kirchner es el que más se acerca a la figura de efectivo coordinador del gabinete. Fernández es abogado y fue superintendente de Seguros durante el gobierno de Menem. No obstante haber pertenecido al PJ, en 2000 postuló a diputado por Buenos Aires en la lista que apoyaba a Domingo Cavallo. Luego volvió al justicialismo, siendo jefe de la campaña de Kirchner, quien luego lo nombró al mando de la JGM.

${ }^{23}$ Esto lo ratificó el mismo Terragno al señalar que "tuve una experiencia muy amarga en los pocos meses que fui jefe de Gabinete, porque a medida que se acentuaban las diferencias había un claro propósito del Presidente de vaciar la Jefatura de Gabinete" (Terragno, 2004).

${ }^{24}$ De hecho, al anunciarse su nombramiento se conoció también que AFIP pasaría a estar bajo dependencia de la jefatura de gabinete. Esto no ocurrió durante la gestión de Terragno, aunque él lo pidió muchas veces. 
La centralidad de Fernández dentro del gabinete debe entenderse considerando las características de ese gobierno. Dado el difícil contexto en que asumió, Kirchner desarrolló desde el inicio una estrategia de "composición del poder y del prestigio presidencial", cuya prioridad era concentrar el poder en el Ejecutivo (Novaro, 2007). Este gobierno se caracterizó entonces por una fuerte preeminencia de Kirchner, constituyéndose un esquema de poder "muy vertical" (entrevista con Mustapic, 2008), en la que el poder del gabinete y de otras instancias fue reducido. El ejercicio del poder se concentró en Kirchner y su círculo íntimo, conocido como "la mesa chica". Ésta la conformaban el propio presidente y su cónyuge Cristina Fernández, Alberto Fernández, el ministro de Planificación Julio de Vido, además del secretario Legal y Técnico, Carlos Zannini. Fuera estaba el ministro de Economía, que hasta noviembre de 2005 fue Roberto Lavagna, quien Kirchner no quería se constituyera en un "superministro" (como fueron varios de sus antecesores), pero al que mantuvo en el cargo mientras lo necesitó, pues fue el artífice de la recuperación económica. La salida de Lavagna, más el hecho de que sus sucesores (Felisa Miceli y Miguel Peirano) fueron débiles políticamente, ratificó la decisión del presidente Kirchner el poder decisorio (también en lo económico) debía centrarse en el presidente y su círculo íntimo (Berenzstein, 2005).

En ese marco, no existían las reuniones de gabinete, respondiendo los ministros directamente a Kirchner (la relación era bilateral) y la coordinación entre ellos era canalizada y controlada por Fernández. Además, fue un efectivo articulador del "kirchnerismo" en el Congreso, implementando una estrategia para generar una bancada leal al presidente, la que no existía en su inicio. El resultado fue positivo, ya que progresivamente pudo quebrar liderazgos internos y crear una base propia de poder, la que se consolidó en 2005 (Godio, 2006; Arzadun, 2008). En el desarrollo de la actividad legislativa, los proyectos eran canalizados por Fernández y los jefes de bancada (Novaro, 2007).

La actividad de Fernández se extendió a otros ámbitos. Por un lado, fue un eficaz comunicador del gobierno, siendo su principal vocero, a diferencia de otros ministros que hablaban poco públicamente (La Nación 24.7.2008). Por el otro, ejerció en nombre de Kirchner las atribuciones que le otorgaban los "superpoderes" 25 . En particular, la

${ }^{25} \mathrm{El}$ otorgamiento al Ejecutivo de facultades especiales en materia de gestión presupuestaria no se inauguró con Kirchner. De hecho, las hubo en los gobiernos de 
JGM ejerció atribuciones relativas a ampliar créditos presupuestarios y determinar su posterior distribución, además de las reestructuraciones del presupuesto ${ }^{26}$.

Fernández se constituyó entonces en una gran "mano derecha" de Kirchner, quien delegó en él grandes responsabilidades, aunque siempre se ocupó de reservar para sí las decisiones últimas. Consecuentemente, si bien Fernández ejerció efectivamente un rol de jefe de gabinete en cuanto era su comunicador, coordinador legislativo y ejecutor de importantes atribuciones en materia presupuestaria (incluso más allá de las que tuvo Economía), no ejerció el cargo en forma "autónoma" de Kirchner, sino como su delegado (entrevistas con Novaro y Orsini, ambas 2008).

\section{Observaciones sobre otros aspectos de la actividad del JGM}

Además de lo dicho en los acápites anteriores, cabe subrayar otros aspectos relacionados con la actividad y funciones de la JGM que refuerzan el argumento de las dificultades que ésta ha tenido para establecerse dentro del sistema.

Una primera observación se refiere a las atribuciones que tiene la JGM respecto de la vinculación Ejecutivo-Congreso (véase Cuadro $\mathrm{N}^{\mathrm{o}}$ 1). En particular, resalta el que, en los hechos, la actividad del jefe de gabinete está lejos de lo que previene la Constitución. Por ejemplo, respecto de la obligación de asistencia mensual al Congreso, la práctica es que salvo Jorge Rodríguez, quien sí asistía periódicamente, los demás ministros han tenido una asistencia muy esporádica (entrevista con Orsini, 2008). De hecho, en 2005, Fernández casi no asistió al par-

Menem, De la Rúa y Duhalde. Sin embargo, las concedidas a partir de 2004 a Kirchner tienen un carácter distinto, pues no se otorgaron en un marco de contracción económica, donde era necesario modificar los presupuestos, sino en uno de fuerte recuperación. Además, en el caso de Kirchner se le autorizó a ejercer estas atribuciones sin sujetarse a la ley de responsabilidad fiscal, cosa que no ocurría antes (Uña, Bertello y Cogliandro, 2004).

${ }^{26}$ De hecho, en uso de estas facultades en 2005 la JGM aumentó el presupuesto en $5,9 \%$, además de realizar reasignaciones por $2,4 \%$. A ello, y en otro aspecto que es fundamental en esta materia, se agrega que varias modificaciones presupuestarias se hicieron a través de los decretos de necesidad y urgencia (DNU): mediante ellos se aumentó el gasto en $14 \%$ en 2005 . Esta situación se produce porque los proyectos de presupuesto subestiman las variables que lo sustentan, de forma tal que en definitiva la recaudación es mayor y se recurre a ese instrumento para gastar el dinero (Díaz, 2006). 
lamento en esa función, a la vez que el ministro Massa sólo asistió una vez a informar sobre la marcha del gobierno en los primeros 8 meses de su gestión (Castells, 2008; La Nación 14.4.2009). En el marco de la relación Ejecutivo-Congreso destaca también que, salvo durante el período de Fernández (que tuvo características particulares), la JGM no se ha asentado como una institución coordinadora de la actividad legislativa del Ejecutivo, y consecuentemente tampoco como la encargada de liderar la construcción de acuerdos entre ambas ramas (entrevista con Orsini, 2008). Esto, sin perjuicio de la labor de seguimiento de la actividad legislativa que realiza ${ }^{27}$, la que no obstante tiene un carácter fundamentalmente informativa.

Un segundo punto relevante se refiere a que la destitución del titular de la JGM podría utilizarse como "fusible" en caso de una crisis institucional de relevancia y así fungir como mecanismo institucional de solución de la misma (contribuyendo así a superar la "rigidez" del sistema). Así fue planteado durante la discusión sobre la reforma, como en el Consejo para la Consolidación de la Democracia (Barraza y Schafrik, 1999: 141-142; Sagües, 2006). Un escenario propicio para que esto ocurriera fue la crítica situación que vivió Argentina en diciembre de 2001, en la que la salida del titular de la JGM (Colombo) y su eventual reemplazo por otra persona (tal vez un opositor) podría haber operado como mecanismo de solución de crisis. Sin embargo, nada de ello ocurrió, toda vez que la destitución de Colombo nunca se planteó seriamente por ningún actor relevante (Serrafero, 2003). Esto, por un lado, porque Colombo era el único ministro que tenía cierto respaldo de la oposición peronista mientras el gobierno se desmoronaba. Por el otro, y más importante, porque en realidad, y dadas las características de la institución, su destitución no resolvía nada concreto, pues en todo caso De la Rúa seguiría siendo el jefe de gobierno y por ende el responsable de la gestión. Incluso en el caso de que se hubiera destituido a Colombo y designado un titular de la JGM que fuera miembro de la oposición peronista, el problema subsistía, ya que, a menos de que De la Rúa hubiera aceptado ser una figura simplemente decorativa y entregara de facto el poder a la oposición (y por una vía no institucional), no habría

${ }^{27}$ Para esos fines dispone de funcionarios (conocidos como "enlaces") que se encargan de monitorear la actividad legislativa que se desarrolla en el Congreso e informarla al Ejecutivo. En octubre de 2008, veinte personas tenían esa función, cada una con la obligación de hacer el seguimiento en comisiones específicas. 
habido un cambio real que permitiera superar la debilidad del gobierno de la Alianza. Dicho de otra forma, el conflicto era entre la oposición y el presidente, donde la primera exigía la salida de De la Rúa como solución, no la de Colombo.

\section{Conclusiones}

Del examen del estatuto jurídico y diversos aspectos del desarrollo institucional de la JGM surgen un conjunto de conclusiones que, por una parte, muestran que en la forma en que está estructurada difícilmente puede constituir un mecanismo de atenuación del presidencialismo, como fue planteado por quienes acordaron la reforma de 1994. Por la otra, que su inserción dentro del sistema institucional aún es incompleta, siendo obstaculizada tanto por elementos institucionales como por decisiones de la autoridad ejecutiva.

En primer lugar, las disposiciones constitucionales que fijan el estatuto de la JGM no importan una modificación del sistema presidencial argentino, en términos de acercarlo a una forma parlamentaria o semipresidencial. Esto, principalmente, porque la reforma de 1994 conservó en manos del presidente, que de acuerdo a la misma es el jefe supremo de la nación y jefe de gobierno, todas sus atribuciones principales. A la vez, buena parte de las atribuciones de la JGM son ejercidas por delegación presidencial; y además la autonomía que pudiera tener (en lo formal) para ejercer algunas atribuciones, es simplemente anulada por la Constitución al permitir que el presidente destituya al titular de la JGM cuando lo estime conveniente. De la misma manera, tampoco ha demostrado ser una institución que permita, en los hechos, un mayor control del Ejecutivo por parte del Congreso. Esto, no sólo porque para su destitución se requiere la mayoría en ambas cámaras, sino también porque si ello ocurriera el presidente puede designar a otra persona en el cargo. Además, puede suceder que el titular de la JGM (como ha ocurrido en muchas ocasiones) no sea un ministro políticamente relevante, y con ello su destitución no tenga un impacto político significativo.

En segundo lugar, la experiencia práctica de la JGM entre 1995 y 2007 pone de manifiesto las dificultades que ha tenido para constituirse en un ministerio que ejerza una labor de coordinación y de liderazgo dentro del gabinete en las materias que se le asignan (por ejemplo, relación con el Congreso, presupuesto). A este respecto, la experiencia 
demuestra que el compromiso de la autoridad política (en este caso el presidente) es fundamental para que una institución se asiente y tome el control efectivo de las funciones que se le asignan. En el caso de la JGM hemos visto como los presidentes han tenido una visión muy diversa acerca del rol que a este ministerio le cabe, lo que se ha traducido en que la JGM ha cumplido una función variable dentro del sistema, cambiándose continuamente algunas de las misiones que se le asignan (y con ello también su estructura institucional); a la vez que no ha logrado tomar el control efectivo de ámbitos que le son propios. En este sentido, la elección de las personas que han ocupado el cargo es fundamental para entender esta situación, ya que sólo en los casos en que el presidente ha designado al frente de la JGM personas de su confianza, la institución ha podido ejercer una función más cercana a lo que establece la Constitución. De esta manera, su influencia como institución ha sido muy variable, teniendo un rol secundario durante buena parte del tiempo, mientras con Kirchner fue efectivamente un centro articulador del gobierno, especialmente gracias a la cercanía de Fernández y Kirchner.

Consecuentemente, la JGM tampoco es un mecanismo que en un sistema presidencial asegure una mejor dirección del gobierno, precisamente porque su titular no tiene la fortaleza institucional para ejercer una posición de liderazgo al interior del gabinete. Ésta la puede obtener en el caso de que el presidente se la asigne, aun cuando para él/ella siempre será posible revocar una decisión en este sentido, y por tanto la posición de la JGM es siempre dependiente de la voluntad presidencial.

En tercer lugar, visto como instrumento para servir de "fusible" ante situaciones de crisis que se pueden generar en los sistemas presidenciales derivadas de su rigidez institucional, tampoco ha sido un mecanismo idóneo. Ello precisamente porque sus características institucionales le impiden serlo: siendo una figura subordinada al presidente, su remoción no altera nada relevante, salvo que al mismo tiempo el jefe de gobierno renuncie de facto a ejercer sus atribuciones. En la crisis de 2001 ello quedó perfectamente claro, porque el conflicto era entre la oposición y el jefe de gobierno (el presidente), y no con un ministro dependiente de él, cuya dimisión no era vista como solución, ni base para un compromiso con la oposición mayoritaria. Para la oposición, ésta era la salida del presidente, no otra. De esta manera, no operó como un mecanismo adecuado para superar la "rigidez" del presidencialismo en 
una situación de crisis, y ni siquiera fue vista como tal por los mismos actores que la crearon.

La experiencia de la JGM debe ser estudiada por los países que en el futuro pretendan modificar su sistema presidencial, ya que demuestra, por una parte, que sin un adecuado dispositivo institucional las intenciones de atenuar el presidencialismo simplemente pueden ser inconducentes. Por el otro, porque la efectiva instalación y asentamiento de una institución no depende sólo de su marco jurídico, sino también de la voluntad política de los gobernantes, que a través de sus decisiones (entre otras cosas, las que se refieren a las personas que ocupen los cargos) pueden afectar negativamente su proceso de inserción dentro del sistema y en definitiva impedir que se constituyan en los instrumentos en los que el sistema institucional pretende.

\section{REFERENCIAS BIBLIOGRÁFICAS}

\section{Entrevistas}

Carlos Acuña, profesor Universidad de San Andrés, octubre de 2008.

Manuel Mora y Araujo, consultor, octubre de 2008.

Ana Mustapic, profesora Universidad Di Tella, octubre 2008.

Marcos Novaro, profesor Universidad de Buenos Aires, octubre 2008.

Paula Orsini, Asesora Secretaria de relaciones parlamentarias, octubre 2008.

Nolberto Perotti, Subsecretario de Evaluación de Presupuesto, octubre de 2008.

Mario Serrafero, profesor universidad UADE, octubre 2008.

\section{Artículos, documentos y libros}

Alfonsín, Raúl: Democracia y Consenso. Buenos Aires: Corregidor, 1996.

Amorim, Octavio: Presidencialismo e Gobernabilidade nas Americas. Rio de Janeiro: KAS-FGV, 2006.

Arzadun, Daniel: El Peronismo: Kirchner y la Conquista del Reino. Buenos Aires: COPPAL, 2008.

Barraza, Javier y Fabiana Schafrik: El Jefe de Gabinete de Ministros. Buenos Aires: Abeledo Perrot, 1999.

Berenzstein, Sergio: "El Hiperpresidencialismo Puede Ahogar la Democracia". www. lanacion.com.ar [29.11.2005].

Berríos, Fabiola y Ricardo Gamboa. "El Congreso Nacional y el Ejercicio de sus Funciones Legislativa y Fiscalizadora”. Revista Politica 47, 2006.

Bidart, Germán: Tratado Elemental de Derecho Constitucional Argentino. Tomo 4. Buenos Aires: EDIAR, 1995.

Carmona, Carlos. "Modificaciones al Órgano Presidencial que Introduce la Ley de Reforma Constitucional No. 20.050”. Revista de Derecho Público 68, 2006.

Castells, Alberto: “Argentina: Figura y Función (Jefatura de Gabinete)”. www.lanacion. com.ar, [29.7.2008]. 
Chasquetti, Daniel: "Democracia, Multipartidismo y Coaliciones en América Latina: Evaluando la Difícil Combinación”. En Jorge Lanzaro (editor), Tipos de Presidencialismo y Coaliciones Políticas en América. Buenos Aires: CLACSO, 2001.

Cheibub, José: Presidentialism, Parliamentarism, and Democracy. New York: Cambridge University Press, 2007.

Cheibub, José y Fernando Limongi: "Democratic Institutions and Regime Survival: Parliamentary and Presidential Democracies Reconsidered”. Annual Review of Political Science 5, 2002.

Díaz, Luciana: "El Debate sobre los Superpoderes". www.cippec.org, Informe Análisis 2, 2006.

Dickinson, Matthew: "Neustadt, New Institutionalism, and Presidential Decision Making: A Theory and Test". Presidential Studies Quarterly 35 (2), 2005.

Dickinson, Mathew y Matthew Lebo: "Reexamining the Growth of the Institutional Presidency, 1940-2000”. http://ms.cc.sunysb.edu/ mlebo/_private/Papers/ Reexamining.pdf, 2002.

De Riz, Liliana y Daniel Sabsay: "El Jefe de Gabinete en el Sistema Presidencial Argentino". En Dieter Nohlen y Mario Fernández (ed.), El Presidencialismo Renovado. Instituciones y Cambio Político en América Latina. Caracas: Nueva Sociedad, 1998.

Duverger, Maurice: "A New Political System Model: Semi-Presidential Government". EJPR 8, 1980.

Eguiguren Praeli, Francisco: "Las Relaciones entre Gobierno y Parlamento: La Particularidad del Régimen Presidencial del Perú". Anuario de Derecho Constitucional Latinoamericano, 2004.

Elgie, Robert: "The Perils of Semi-Presidentialism. Are They Exaggerated?" Democratization 15 (1), 2008.

Fernández Meijide, Graciela: La Ilusión. El Fracaso de la Alianza visto por Dentro. Buenos Aires: Editorial Sudamericana, 2007.

Foweraker, Joe: "Review Article: Institutional Design, Party Systems and GovernabilityDifferentiating the Presidential Regimes of Latin America". British Journal of Political Science 28, 1998.

García Lema, Alberto Manuel: La Reforma por Dentro. Buenos Aires: Planeta, 1994.

Godio, Julio: El Tiempo de Kirchner. Buenos Aires: Letragrifa, 2006.

Godoy, Óscar (ed.): Cambio de Régimen Politico. Santiago: Ediciones Universidad Católica de Chile, 1992.

Huneeus, Carlos: "Por qué Ha Funcionado el Presidencialismo en Chile". Persona y Sociedad XIX (2) 2005.

Jones, Mark: "Presidential Election Laws and Multipartism in Latin America". Political Research Quarterly 47 (1), 1995.

"Evaluating Argentina's Democracy". En Mainwaring, Scott y Matthew Shugart (editores), Presidentialism and Democracy in Latin America. Cambridge: Cambridge University Press, 1997.

Kessell, John: "The Structures of the Reagan White House". American Journal of Political Science 28, (2), 1984.

Leiras, Marcelo: Todos los Caballos del Rey. La Integración de los Partidos Políticos el Gobierno Democrático de la Argentina, 1995-2003. Buenos Aires: Prometeo, 2007. 
Linz, Juan: "Democracia: Presidencialismo o Parlamentarismo. ¿Hace alguna Diferencia?”. En Juan Linz, Arend Lijphart, Arturo Valenzuela y Óscar Godoy (eds.), Hacia una Democracia Moderna. La Opción Parlamentaria. Santiago: PUC, 1990.

Mainwaring, Scott: "Presidentialism, Multipartism, and Democracy: The Difficult Combination". Comparative Political Studies 26 (2), 1993.

Mainwaring, Scott y Matthew Shugart: "Juan Linz: Presidencialismo y Democracia. Una Revisión Crítica”. Desarrollo Económico 135, 1994.

Martínez Gallardo, Cecilia. "Designing Cabinets: Presidents, Politics and Policymaking in Latin America". Tesis Doctoral, University of Columbia, 2005.

Metcalf, Lee K: "Measuring Presidential Power." Comparative Political Studies 33, 2000.

Morgenstern, Scott y Benito Nacif (editores): Legislative Politics in Latin America. Cambridge: Cambridge University Press, 2002.

Munck, Gerardo: "Democratic Politics in Latin America: New Debates and Research Frontiers". Annual Review of Political Science 7, 2004.

Mustapic, Ana: "Oscillating Relations: President and Congress in Argentina”. En Scott Morgenstern y Benito Nacif (editores), Legislative Politics in Latin America. Cambridge: Cambridge University Press, 2002.

Negretto, Gabriel: "Procesos Constituyentes y Distribución de Poder: La Reforma del Presidencialismo Argentino". Politica y Gobierno 3 (1), 2001.

- "Paradojas de la Reforma Constitucional en América Latina". Journal of Democracy en Español 1, 2009.

Nino, Carlos: "Presidencialismo vs. Parlamentarismo". En Consejo Para la Consolidación de la Democracia, Presidencialismo vs. Parlamentarismo: Materiales para el Estudio de la Reforma Constitucional. Buenos Aires: Eudeba, 1988.

Nolte, Detlef: "Presidentialism Revisited: Gewaltentrennung und Gewaltverschränkung in den Lateinamerikanischen Prasidialdemokratien". Lateinamerika Analysen 7, 2004.

Novaro, Marcos: "La Jefatura de Gabinete y sus Capacidades de Coordinación y Control". Buenos Aires, 2001, Mimeo.

"El Presidencialismo Argentino entre el Derrumbe de 2001 y la Recomposición Actual". Buenos Aires, 2007 (Mimeo).

Pasquino, Gianfranco: Los Poderes de los Jefes de Gobierno. Buenos Aires: Prometeo, 2007.

Payne, Mark: "Balancing Executive and Legislative Prerogatives: The Role of Constitutional and Party-Based factors". En Mark Payne, Daniel Zovatto y Mercedes Díaz, Democracies in Development. Washington: IADB, 2007.

Pérez-Liñán, Aníbal: "Pugna de Poderes y Crisis de Gobernabilidad ¿Hacia un Nuevo Presidencialismo?" Latin American Research Review 38 (3), 2003.

Quiroga, Hugo: La Argentina en Emergencia Permanente. Buenos Aires: Eudeba, 2005.

Roncangliolo, Rafael: "El Primer Ministro en Perú: ¿Institución o Retórica? En Dieter Nohlen y Mario Fernández (editores), Presidencialismo v/s Parlamentarismo. América Latina. Caracas: Nueva Sociedad, 1991.

Sagües, Néstor: "Parlamentarismo y Presidencialismo. Un Ensayo Sistémico para la Gobernabilidad Democrática: El "Minipremier" en Perú y Argentina. Experiencias y Perspectivas”. En Carrasco, Pérez, Urías, Terol (coordinadores), 
Derecho Constitucional para el Siglo XXI: Actas del VIII Congreso Iberoamericano de Derecho Constitucional, Vol. 2, 2006.

Sartori, Giovanni: Ingeniería Constitucional Comparada. México: FCE, 1994.

San Martino de Dromi, María: Argentina Contemporánea, de Perón a Menem. Buenos Aires: Ediciones Ciudad Argentina, 1996.

Serrafero, Mario: "La Jefatura de Gabinete y las Crisis Políticas: El Caso De la Rúa". Revista SAAP, Vol. 1 (3), 2003.

Shugart, Matthew y John Carey: Presidents and Assemblies. Constitutional Design and Electoral Dynamics. Cambridge: Cambridge University Press, 1992.

Siavelis, Peter: The President and Congress in Postauthoritarian Chile. Institutional Constraints to Democratic Consolidation. University Park: Pennsylvania State University Press, 2000.

Terragno, Rodolfo. Entrevista en revista Noticias. www.terragno.gov.ar, 2004.

Thibaut, Bernhard: Präsidentialismus und Demokratie in Lateinamerika: Argentinien, Brasilien, Chile und Uruguay im historischen Vergleich. Leske+Budrich: Opladen, 1996.

Uña, Gerardo, Nicolás Bertello y Gisell Cogliandro: "Delegación de Facultades al Jefe de Gabinete de Ministros: Evolución e Impacto en el Contexto Fiscal Actual". En http://129.3.20.41/eps/pe/papers/0505/0505011.pdf (Documento preparado para Fundación Konrad Adenauer.), 2004.

Valenzuela, Arturo: "Partidos Políticos y Crisis Presidencial en Chile: Proposición para un Gobierno Parlamentario". En Juan Linz, Arend Lijphart, Arturo Valenzuela y Óscar Godoy (eds.), Hacia una Democracia Moderna. La Opción Parlamentaria. Santiago: PUC, 1990. 